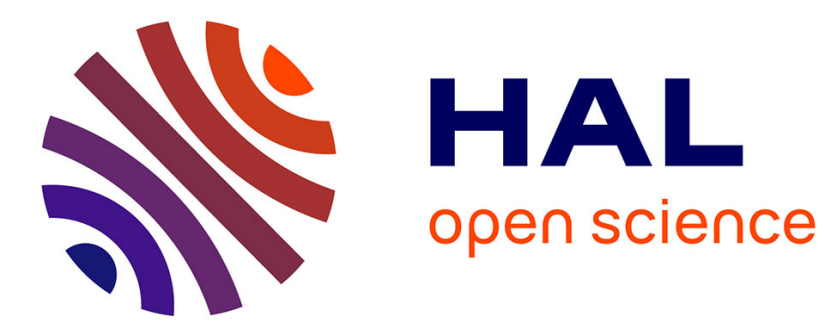

\title{
Controlling the occupation time of an exponential martingale
}

Stefan Ankirchner, Christophette Blanchet-Scalliet, Monique Jeanblanc

\section{To cite this version:}

Stefan Ankirchner, Christophette Blanchet-Scalliet, Monique Jeanblanc. Controlling the occupation time of an exponential martingale. Applied Mathematics and Optimization, 2017, 76 (2), pp.415-428. 10.1007/s00245-016-9356-2 . hal-01227899v2

\section{HAL Id: hal-01227899 \\ https://hal.science/hal-01227899v2}

Submitted on 8 Apr 2016

HAL is a multi-disciplinary open access archive for the deposit and dissemination of scientific research documents, whether they are published or not. The documents may come from teaching and research institutions in France or abroad, or from public or private research centers.
L'archive ouverte pluridisciplinaire HAL, est destinée au dépôt et à la diffusion de documents scientifiques de niveau recherche, publiés ou non, émanant des établissements d'enseignement et de recherche français ou étrangers, des laboratoires publics ou privés. 


\title{
Controlling the occupation time of an exponential martingale
}

\author{
Stefan Ankirchner* Christophette Blanchet-Scalliet ${ }^{\dagger}$ \\ Monique Jeanblanc $\ddagger$
}

March 24, 2016

We consider the problem of maximizing the expected amount of time an exponential martingale spends above a constant threshold up to a finite time horizon. We assume that at any time the volatility of the martingale can be chosen to take any value between $\sigma_{1}$ and $\sigma_{2}$, where $0<\sigma_{1}<\sigma_{2}$. The optimal control consists in choosing the minimal volatility $\sigma_{1}$ when the process is above the threshold, and the maximal volatility if it is below. We give a rigorous proof using classical verification and provide integral formulas for the maximal expected occupation time above the threshold.

\section{Introduction}

Let $X^{\alpha}$ be an exponential martingale with controlled dynamics $d X_{t}^{\alpha}=\alpha_{t} X_{t}^{\alpha} d W_{t}$, where $W$ is a one-dimensional Brownian motion. Consider the problem of maximizing the

\footnotetext{
*Stefan Ankirchner, Institute for Mathematics, University of Jena, Ernst-Abbe-Platz 2, 07745 Jena, Germany. Email: s.ankirchner@uni-jena.de, Phone: +49 (0)3641 946275.

${ }^{\dagger}$ Christophette Blanchet-Scalliet, Université de Lyon - CNRS, UMR 5208, Institut Camille Jordan - Ecole Centrale de Lyon, 36 avenue Guy de Collongue, 69134 Ecully Cedex, FRANCE. Email: christophette.blanchet@ec-lyon.fr, Phone:+33472186405

${ }^{\ddagger}$ Monique Jeanblanc, Laboratoire de Mathématiques et Modélisation d’Évry, (LaMME), Université d'Evry Val d'Essonne, UMR CNRS 8071, Evry, France, Email: monique.jeanblanc@univ-evry.fr S.A. thanks the Ecole Centrale de Lyon for the kind invitation in May 2015, and the University of Jena for granting a sabbatical leave in the summer 2015.

M.J. thanks Jena University for the fruitful invitation in March 2015. The research of M.J. is supported by the Chaire Marchés en Mutation (Fédération Bancaire Française).

C.B. thanks Jena University for the invitation in July 2015.

We are grateful to Ingo Althöfer for drawing our attention to the topic of diffusion control. Moreover, we thank seminar participants at METU Ankara and the University of Duisburg-Essen for helpful comments.
} 
expected amount of time the process $X^{\alpha}$ spends above a positive constant threshold up to a finite time horizon. Assume that one can choose as control any predictable process $\alpha$ that takes values between two constants $\sigma_{1}$ and $\sigma_{2}$, where $0<\sigma_{1}<\sigma_{2}$.

The optimal control is of bang-bang type and consists in choosing the minimal volatility $\sigma_{1}$ when the process is above the threshold (winning region), and to choose the maximal volatility $\sigma_{2}$ when the process is below the threshold (loosing region). This is intuitively plausible, because the volatility determines the speed of the diffusion process and hence the exit time from the winning resp. loosing region. Choosing the maximal speed in the loosing region minimizes the return time to the winning region, provided the process does return; and choosing the minimal speed in the winning region maximizes the return time to the loosing region.

The aim of the paper is to rigorously prove that the bang-bang control is optimal and to quantify the expected time the optimally controlled martingale spends above the threshold. To this end we compute the Laplace transform of the expected occupation time above the threshold, considered as a function of time. We show that an inversion integral formula applies for the density of the occupation time. This allows to show smoothness of the candidate value function and to perform a classical verification.

The control problem considered here gathers in stylized form the situation of a manager who can control the volatility of a firm's value process. Suppose that the manager gets bonus payments at a constant rate whenever the value process performs better than a reference index. Thus the manager aims to maximize the expected time the value process spends above the index. The optimal control affirms that such a bonus scheme incentivizes the manager to choose risky strategies when the firm's value is underperforming and to play safe in case if it performs well. Our quantitative results show that the increase in the occupation time in the winning region can be considerable in the short run (see Figure 1). We stress that, for maximizing the expected bonus, during underperforming periods it is optimal to choose the maximal volatility, even if this does not entail a higher drift.

The corresponding control problem within an arithmetic Brownian motion, i.e., the case where $d Z_{t}=\sigma_{t} d W_{t}$ is the controlled process, has received some attention in the literature. Consider the particular feedback control $\sigma(z)=\sigma_{1}$ for $z \geq 0$ and $\sigma(z)=\sigma_{2}$ for $z<0$. McNamara [7] analyzes for which terminal reward functions this feedback control $\sigma$ is optimal among all controls valued in $\left[\sigma_{1}, \sigma_{2}\right]$. In particular, his results imply that $\sigma$ maximizes the expected occupation time of the controlled Brownian motion above zero, and that the maximal expected occupation time up to time $T$ is given by $\frac{\sigma_{2}}{\sigma_{1}+\sigma_{2}} T$ (cf. Remark 8, [7]). Keilson and Wellner [6] compute the transition probability of the optimally controlled process $Z$ satisfying $d Z_{t}=\sigma\left(Z_{t}\right) d W_{t}$. Their approach relies on the symmetry of the transition probability, and, therefore, seems to be difficult to adapt to the geometric case. Finally, Althöfer et al. [1] analyze the bang-bang control $\sigma$ within discrete-time random walk models. They use combinatorial methods to determine the limiting probability for the processes to be in the winning region. 


\section{Controlling the occupation time above the level 1}

Let $W$ be a Brownian motion on a probability space $(\Omega, \mathcal{F}, \mathbb{P})$ and denote by $\left(\mathcal{F}_{t}\right)_{t \geq 0}$ its augmented natural filtration. Let $\mathcal{A}$ be the set of $\left(\mathcal{F}_{t}\right)$-progressively measurable processes with values in $\left[\sigma_{1}, \sigma_{2}\right]$, where $0<\sigma_{1}<\sigma_{2}$. For $\alpha \in \mathcal{A}$ and $x>0$, let $X^{x, \alpha}$ be the solution of the SDE

$$
d X_{t}^{x, \alpha}=\alpha_{t} X_{t}^{x, \alpha} d W_{t}, \quad X_{0}^{x, \alpha}=x .
$$

Note that, due to $0<\alpha \leq \sigma_{2}, X^{\alpha}$ is a positive process. We denote by

$$
K(t, x, \alpha)=\int_{0}^{t} 1_{\left\{X_{s}^{x, \alpha} \geq 1\right\}} d s, \quad t \in[0, \infty],
$$

the occupation time of $X^{x, \alpha}$ above 1 up to time $t$. We consider the problem of maximizing the expected occupation time. To this end, we introduce the value function

$$
v(t, x)=\sup _{\alpha \in \mathcal{A}} E[K(t, x, \alpha)]=\sup _{\alpha \in \mathcal{A}} \int_{0}^{t} P\left(X_{s}^{x, \alpha} \geq 1\right) d s, \quad t \in[0, \infty) .
$$

We show that the strategy in $\mathcal{A}$ maximizing the occupation time above 1 up to any finite time horizon $t$ is a feedback control of bang-bang type. As usual, we say that a strategy $\alpha$ is a feedback control if there exists a function $\hat{\alpha}: \mathbb{R}_{+} \rightarrow\left[\sigma_{1}, \sigma_{2}\right]$ such that $\alpha_{t}=\hat{\alpha}\left(X_{t}^{x, \hat{\alpha}}\right)$, where $X^{x, \hat{\alpha}}$ is the solution of the $\mathrm{SDE}$

$$
d X_{t}=\hat{\alpha}\left(X_{t}\right) X_{t} d W_{t}, \quad X_{0}=x
$$

One can show that for any measurable function $\hat{\alpha}$ with values in $\left[\sigma_{1}, \sigma_{2}\right]$ and of bounded variation on any compact interval the $\operatorname{SDE}(2)$ has a unique strong solution. Indeed, due to $\alpha \in\left[\sigma_{1}, \sigma_{2}\right]$, the solution, if it exists, is positive. So $\left(X_{t}\right)$ solves (2) if and only if $\log \left(X_{t}\right)$ satisfies the $\mathrm{SDE}$

$$
d Z_{t}=\hat{\alpha}\left(e^{Z_{t}}\right) d W_{t}-\frac{1}{2} \hat{\alpha}\left(e^{Z_{t}}\right)^{2} d t, \quad Z_{0}=\log (x) .
$$

The results in [8] guarantee pathwise uniqueness for the SDE (3). Together with weak existence this further implies the existence of a strong solution of (3) (cf. Section 5.3, [5]). Consequently, (2) possesses a unique strong solution.

For simplicity in the following we do not distinguish between the function $\hat{\alpha}$ and the associated feedback control $\alpha$.

We denote by $\eta$ the feedback control that consists in choosing the lowest volatility if the process $X$ is greater than or equal to one, and the highest volatility if $X$ is below one. More precisely, let

$$
\eta(x)= \begin{cases}\sigma_{1}, & x \geq 1 \\ \sigma_{2}, & x<1\end{cases}
$$

The aim of the paper is to prove that $\eta$ maximizes the occupation time above 1 and to compute the value function, i.e. the maximal expected occupation time. It turns 
out to be difficult to determine the value function explicitly. Instead, we give a closed form expression for $\mathcal{L}[v(\cdot, x)](\lambda)=\int_{0}^{\infty} e^{-\lambda t} v(t, x) d t$, the Laplace transform of the value function considered as a function of $t$. The following theorem is our main result, the proof of which is postponed to Section 4 .

Theorem 1.1. The unique optimal control for problem (1) is given by the function $\eta$, defined in (4). The Laplace transform of $t \mapsto v(t, x)$ satisfies, for $\lambda>0$,

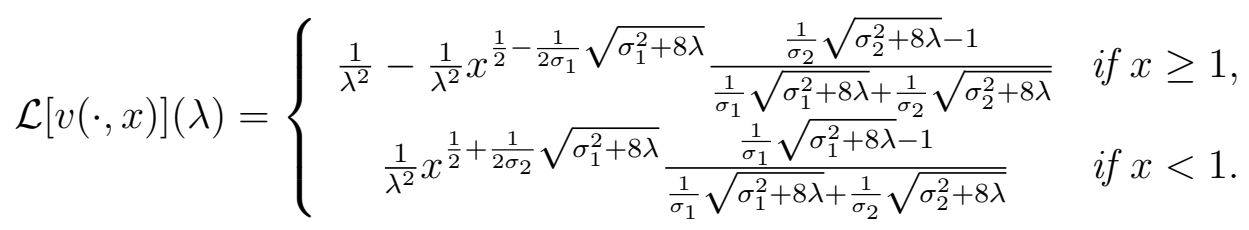

To give an intuition for why $\eta$ is optimal, notice that one can interpret $X$ as timechanged geometric Brownian motion (GBM). The control $\alpha$ determines the time change, and hence the distribution of the process' occupation time above 1. By choosing the strategy $\eta$, the occupation time is concentrated closer to the origin. We remark, however, that the expected total occupation time $E(K(\infty, x, \alpha))$ does not depend on the control values chosen when the process $X$ is below 1 (see remark 2.2).

In the arithmetic case one can show that the strategy maximizing the expected occupation time up to a time point $T$ coincides with the control maximizing the probability for $X$ to be above 1 at $T$. In the geometric case, $\eta$ does not maximize the probability $P\left(X_{T}^{x, \alpha} \geq 1\right)$ for a given time horizon $T$. The reason is that a high volatility entails $X_{t}^{x, \alpha}$, in average, to attain the level 1 earlier, but also to converge faster to zero as $t \rightarrow \infty$. Therefore, for large time horizons $T$ it is better to choose a small volatility, even when the process is below 1 .

In order to prove Theorem 1.1, we explicitly determine the Laplace transform in $t$ of the function $Q$, defined by

$$
Q(t, x):=P\left(X_{t}^{x, \eta} \geq 1\right) .
$$

The Laplace transform can be identified with the value function of a control problem with an infinite time horizon. This is shown in Section 2. In Section 3 we prove that the function $Q(t, \cdot)$ is twice continuously differentiable in $x$ for $x \neq 1$, and once continuously differentiable for $x=1$. The regularity will allow us to use a verification argument for proving that, for any $t$, the control $\eta$ maximizes $E(K(t, x, \alpha))$ among all $\alpha \in \mathcal{A}$ (see Section 4).

We close this section by explaining how one can use the Laplace transform (5) for calculating the maximal expected occupation time. We do not know the Laplace inverse of (5) explicitly. For $x=1$, however, we obtain a simple integral inversion formula for the function $g(t):=Q(t, 1)$ (see Equation (17)). For arbitrary $x>0$, the function $t \mapsto Q(t, x)$ can be recovered as a convolution of $g$ and the hitting time distribution of the geometric Brownian motion. As a result, we obtain explicit integral formulas for the maximal expected occupation time. The integrals can be evaluated numerically, and hence we can use them for estimating the increase in the expected occupation time by switching from a constant control to the optimal control $\eta$. 
Let us compare the optimal control with the constant control $\hat{\alpha} \equiv \sigma_{1}$. One can show that $\lim _{t \downarrow 0} g(t)=\sigma_{2} /\left(\sigma_{1}+\sigma_{2}\right)$, and $\lim _{t \downarrow 0} P\left(X_{t}^{1, \hat{\alpha}} \geq 1\right)=1 / 2$. Therefore, in the short run, $\eta$ entails a considerably higher occupation time than $\hat{\alpha}$. In the long run, however, $P\left(X_{t}^{1, \hat{\alpha}} \geq 1\right)$ exceeds $g(t)$, and a straightforward computation entails $E(K(\infty, 1, \eta))=E(K(\infty, 1, \hat{\alpha}))=2 / \sigma_{1}$. This shows that the advantage of $\eta$ over $\hat{\alpha}$ vanishes as the time horizon converges to infinity. All these observations are illustrated in Figure 1 for the values $\sigma_{1}=1$ and $\sigma_{2}=2$.
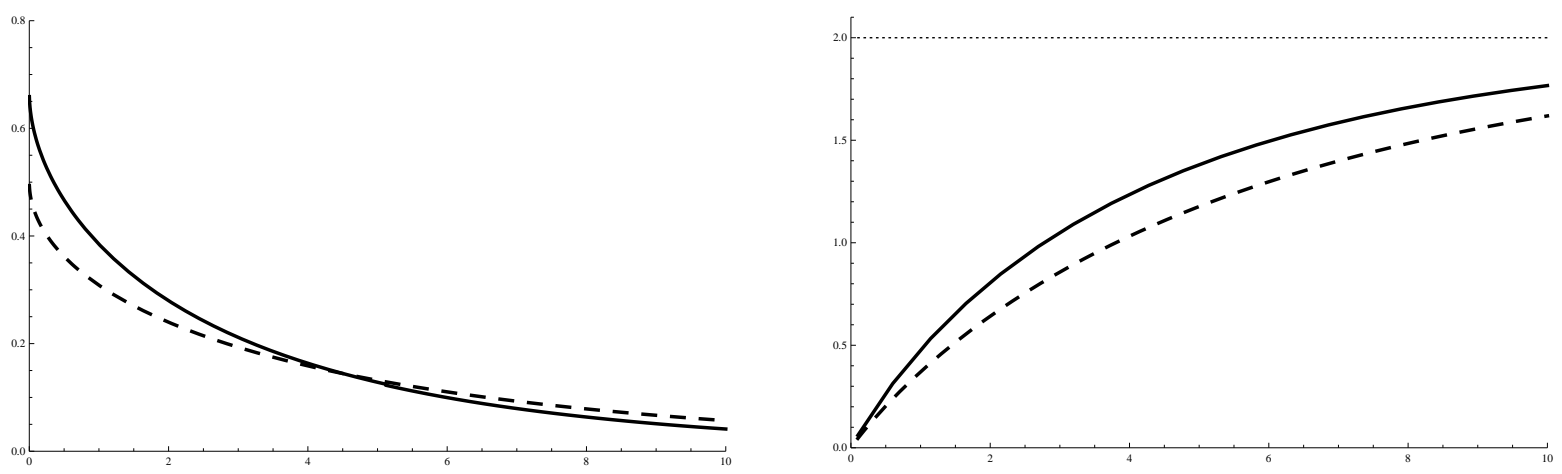

Figure 1: The left-hand side depicts the graphs of $g$ (solid line) and $t \mapsto P\left(X_{t}^{1, \hat{\alpha}} \geq 1\right)$ (dashed line), with $\sigma_{1}=1$ and $\sigma_{2}=2$. The right-hand side shows the corresponding expected occupation time. Note that for both strategies the values converge to 2 , the maximal expected occupation time up to infinity.

\section{A control problem with infinite time horizon}

For any control $\alpha \in \mathcal{A}$ we consider the gain function

$$
J(x, \alpha ; \lambda)=\int_{0}^{\infty} e^{-\lambda t} P\left(X_{t}^{x, \alpha} \geq 1\right) d t
$$

with $\lambda>0$, and we define the associated value function by

$$
V(\lambda, x)=\sup _{\alpha \in \mathcal{A}} J(x, \alpha ; \lambda) .
$$

If there is no ambiguity, we shall write $V(x)$ for $V(\lambda, x)$. The HJB equation of the control problem (7) is given by

$$
-\lambda V(x)+1_{[1, \infty)}(x)+\frac{1}{2} x^{2} \sup _{u \in\left[\sigma_{1}, \sigma_{2}\right]} u^{2} V_{x x}(x)=0 .
$$

We start by heuristic arguments to derive a closed form expression of the value function. Assume that $\eta$ is optimal and that the value function $V$ satisfies the HJB equation. 
Then, since $\lambda V \leq 1$, this yields that $V$ is concave on $[1, \infty[$ and convex otherwise, and that $V$ is a solution of the following equations

$$
\begin{aligned}
& -\lambda V(x)+1+\frac{1}{2} \sigma_{1}^{2} x^{2} V_{x x}(x)=0, \quad x>1, \\
& -\lambda V(x)+\frac{1}{2} \sigma_{2}^{2} x^{2} V_{x x}(x)=0, \quad x \in(0,1) .
\end{aligned}
$$

Notice that the function $V(x)=1 / \lambda$ is a particular solution of (8) and $V(x)=0$ is a particular solution of (9). For $i=1,2$ let

$$
\gamma_{i}=\frac{1}{2}-\frac{1}{2 \sigma_{i}} \sqrt{\sigma_{i}^{2}+8 \lambda}, \text { and } \beta_{i}=\frac{1}{2}+\frac{1}{2 \sigma_{i}} \sqrt{\sigma_{i}^{2}+8 \lambda}
$$

Observe that $\gamma_{i}<0<1<\beta_{i}$, and that $x^{\beta_{1}}$ and $x^{\gamma_{1}}$ are linearly independent solutions of the homogeneous equation associated to (8). Therefore, a solution of (8) has the form $G_{1}(x)=\frac{1}{\lambda}+A_{1} x^{\beta_{1}}+B_{1} x^{\gamma_{1}}$. Similarly, any solution of (9) must satisfy $G_{2}(x)=$ $A_{2} x^{\beta_{2}}+B_{2} x^{\gamma_{2}}$. Since the value function $V$ is bounded, we can match it with $G_{1}$ only if $A_{1}=0$, and with $G_{2}$ only if $B_{2}=0$.

If $V$ is continuously differentiable at $x=1$, then it must hold true that $\frac{1}{\lambda}+B_{1}=A_{2}$ and $\gamma_{1} B_{1}=\beta_{2} A_{2}$. This means

$$
B_{1}=-\frac{1}{\lambda} \frac{1+\sqrt{1+\frac{8 \lambda}{\sigma_{2}^{2}}}}{\sqrt{1+\frac{8 \lambda}{\sigma_{1}^{2}}}+\sqrt{1+\frac{8 \lambda}{\sigma_{2}^{2}}}} \text { and } A_{2}=\frac{1}{\lambda} \frac{\sqrt{1+\frac{8 \lambda}{\sigma_{1}^{2}}}-1}{\sqrt{1+\frac{8 \lambda}{\sigma_{1}^{2}}}+\sqrt{1+\frac{8 \lambda}{\sigma_{2}^{2}}}}
$$

Our heuristic considerations indicate that the value function $V$ is equal to the function $G$ defined by

$$
G(x)= \begin{cases}\frac{1}{\lambda}+B_{1} x^{\gamma_{1}}, & x \geq 1 \\ A_{2} x^{\beta_{2}}, & x<1\end{cases}
$$

(whenever we want to stress the dependence on $\lambda$ we write $G(\lambda, x)$ ).

We next show, via classical verification, that indeed both functions coincide. Notice that $G \in \mathcal{C}^{1}\left(\mathbb{R}_{+}\right) \cap \mathcal{C}^{2}\left(\mathbb{R}_{+} \backslash\{1\}\right)$, but $G$ is not twice continuously differentiable at $x=1$.

Proposition 2.1. We have $V(x)=G(x)$ and $\eta$ is the unique optimal control for problem (7).

Proof. Let $\alpha \in \mathcal{A}$ be an arbitrary control and $X=X^{x, \alpha}$ the associated controlled state process. The Itô formula for functions in $\mathcal{C}^{1}$ with absolutely continuous derivatives implies (see Section 3.7 in [5])

$$
e^{-\lambda t} G\left(X_{t}\right)=G(x)+\int_{0}^{t} e^{-\lambda s} G^{\prime}\left(X_{s}\right) d X_{s}+\int_{0}^{t} e^{-\lambda s}\left(\frac{1}{2} X_{s}^{2} \alpha_{s}^{2} G^{\prime \prime}\left(X_{s}\right)-\lambda G\left(X_{s}\right)\right) d s .
$$

Since $B_{1}<0<A_{2}$ and $\gamma_{1}<0<1<\beta_{2}$, the function $G$ is concave on $(1, \infty)$ and convex on $(0,1)$. It follows that one has $\frac{1}{2} \alpha_{s}^{2} X_{s}^{2} G^{\prime \prime}\left(X_{s}\right)-\lambda G\left(X_{s}\right) \leq-f\left(X_{s}\right)$, where $f(x)=1_{x \geq 1}$. 
By taking expectations, and localizing the local martingale part if necessary, we arrive at

$$
E\left[e^{-\lambda t} G\left(X_{t}\right)\right] \leq G(x)-E \int_{0}^{t} e^{-\lambda s} f\left(X_{s}\right) d s .
$$

The function $|G|$ being bounded by $A_{2}+\frac{1}{\lambda}-B_{1}$, dominated convergence implies that the left-hand side of (11) converges to zero as $t \rightarrow \infty$. Moreover, the right-hand side converges by monotone convergence. Therefore, $G(x) \geq E \int_{0}^{\infty} e^{-\lambda s} f\left(X_{s}\right) d s$. Since $\alpha$ is arbitrary, this entails $G(x) \geq V(x)$.

By choosing the strategy $\alpha=\eta$, we obtain an equality in (11). This further implies that $J(x, \eta)=G(x)$, and hence $G(x) \leq V(x)$.

Finally, observe that $\eta$ is the only control, up to null sets, for which we have equality in (11). Hence $\eta$ is the unique optimal control.

Remark 2.2. If $\lambda=0$, then the value function (7) is still finite. In this case, the HJB equation is written as

$$
\begin{array}{r}
1+\frac{1}{2} x^{2} \sup _{u \in\left[\sigma_{1}, \sigma_{2}\right]} u^{2} V_{x x}(x)=0, \quad x>1, \\
V_{x x}(x)=0, \quad x \in(0,1) .
\end{array}
$$

A verification similar to the one in the proof of Proposition 2.1 shows that the value function is given by

$$
V(0, x)=\left\{\begin{array}{cl}
\frac{2}{\sigma_{1}^{2}}(1+\ln (x)), & x \geq 1, \\
\frac{2}{\sigma_{1}^{2}} x, & x<1 .
\end{array}\right.
$$

In this case, however, $\eta$ is not the only optimal control. Indeed, any control is optimal if it is equal to $\sigma_{1}$ whenever $X_{t} \geq 1$. The values of the control when $X_{t}<1$ determine the speed of the process below the level 1 , and hence the times at which the process attains again the level 1 . However, the total amount spent above 1 is only determined by the process' speed above 1 , and it is maximal if the minimal speed $\sigma_{1}$ is chosen.

Remark 2.3. A similar infinite time horizon problem within an arithmetic Brownian model is considered in Example (7.6) in [10].

Recall that $Q(t, x)=P\left(X_{t}^{x, \eta} \geq 1\right)$. Proposition 2.1 immediately implies that $\lambda \mapsto$ $G(\lambda, x)$ is the Laplace transform of $t \mapsto Q(t, x)$.

Corollary 2.4. The Laplace transform of $Q(\cdot, x)$ is given by

$$
\mathcal{L}[Q(\cdot, x)](\lambda)=G(\lambda, x) .
$$

Notice that the Laplace transform $\mathcal{L}[Q(\cdot, x)](\lambda)$ is continuous in the parameter $x$, for all $x \in(0, \infty)$. This does not directly imply that $Q(t, x)$ is also continuous in $x$. Indeed, the continuity of Laplace transforms in a parameter does not necessarily imply that the original functions are also continuous w.r.t. the parameter (consider e.g. the function family $\left.f(t, x)=1_{[x, \infty]}(t), t, x \in(0, \infty)\right)$. Nevertheless, we have that $Q(t, x)$ is continuous in $x$, as is shown in Proposition 3.4 of the next section. 


\section{Properties of the occupation time density}

In this section we first derive an integral expression for $Q$ by using a Laplace inversion of $G$ for $x=1$.

Let $\tau(x)=\inf \left\{s \geq 0: X_{s}^{x, \eta}=1\right\}$. Up to $\tau(x)$ the process $X^{x, \eta}$ is an exponential martingale either with volatility $\sigma_{1}$ or $\sigma_{2}$. Thus the hitting time distribution can be derived from the hitting time distribution of a standard GBM with constant volatility. Let $H(x, t)=P(\tau(x) \leq t), t \geq 0$, be the cumulative distribution function of $\tau(x)$. For $x \in(0,1)$ we have

$$
H(x, t)=\Phi\left(\frac{\frac{1}{\sigma_{2}} \ln (x)-\sigma_{2} t / 2}{\sqrt{t}}\right)+x \Phi\left(\frac{\frac{1}{\sigma_{2}} \ln (x)+\sigma_{2} t / 2}{\sqrt{t}}\right),
$$

and for $x>1$

$$
H(x, t)=\Phi\left(\frac{-\frac{1}{\sigma_{1}} \ln (x)+\sigma_{1} t / 2}{\sqrt{t}}\right)+x \Phi\left(\frac{-\frac{1}{\sigma_{1}} \ln (x)-\sigma_{1} t / 2}{\sqrt{t}}\right),
$$

(see Section 3.3 in [4]). We denote by $H_{t}$ the derivative of the function $H$ w.r.t. the second variable, i.e., $H_{t}(x, \cdot)$ is the density of $\tau(x)$.

We now introduce $g(t):=Q(t, 1)$ and $c:=\frac{\sigma_{2}}{\sigma_{1}+\sigma_{2}}$. The Markov property of $X^{x, \eta}$ implies that, for any time $t>0$ and for $x<1$

$$
Q(t, x)=E\left[1_{\{\tau(x) \leq t\}} g(t-\tau(x))\right]=\int_{0}^{t} g(t-u) H_{t}(x, u) d u,
$$

and for $x>1$,

$$
Q(t, x)=E\left[1_{\{\tau(x)>t\}}+1_{\{\tau(x) \leq t\}} g(t-\tau(x))\right]=1-H(x, t)+\int_{0}^{t} g(t-u) H_{t}(x, u) d u .
$$

Therefore, for computing $Q$ it only remains to determine $g$. To do so, we introduce some further definitions. Let $D=\left\{z \in \mathbb{C} \mid \operatorname{Re}(z)>-\frac{\sigma_{1}^{2}}{8}\right\}$, and let

$$
F(z)=\frac{1}{z} \frac{\sqrt{1+\frac{8 z}{\sigma_{1}^{2}}}-1}{\sqrt{1+\frac{8 z}{\sigma_{1}^{2}}}+\sqrt{1+\frac{8 z}{\sigma_{2}^{2}}}} .
$$

Note that $F$ is holomorphic on $D$, and that for $\beta>\frac{-\sigma_{1}^{2}}{8}$ we have $\lim _{n \rightarrow \infty} \sup \{|F(z)|$ : $z \in \mathbb{C}$ with $\operatorname{Re}(z) \geq \beta,|z| \geq n\}=0$.

Lemma 3.1. The function $g$ satisfies

$$
g(t)=\lim _{n \rightarrow \infty} \frac{2}{\pi} \int_{0}^{n} \operatorname{Re}(F(i s)) \cos (t s) d s
$$

for all $t>0$. 
Proof. 1. Notice that Corollary 2.4 implies that the Laplace transform of $g$ satisfies $\mathcal{L}[g](\lambda)=F(\lambda)$, for $\lambda>0$.

2. Note that the mapping $\mathbb{R} \ni t \mapsto F(i t)$ is square integrable. Indeed, the function $F$ is bounded on $i \mathbb{R}$ and, for $|t|$ large enough, $|F(i t)|$ is bounded by $\frac{K}{|t|}$ for some constant $K$. Therefore, its inverse Fourier transform is defined, and it is given by the $L^{2}(\mathbb{R})$-limit

$$
\hat{g}(t)=\lim _{n \rightarrow \infty} \frac{1}{2 \pi} \int_{-n}^{n} F(i s) e^{i t s} d s, \quad t \in \mathbb{R},
$$

(see e.g. Thm 9.13 in [11]). We next show that $\hat{g}(t)=g(t)$, for $t>0$. To this end let $\hat{g}_{n}(t)=\frac{1}{2 \pi} \int_{-n}^{n} F(i s) e^{i t s} d s$. Since $\hat{g}_{n}$ converges to $\hat{g}$ in $L^{2}(\mathbb{R})$, we have $\lim _{n} \mathcal{L}\left[\hat{g}_{n}\right](\lambda)=$ $\mathcal{L}[\hat{g}](\lambda)$, for all $\lambda>0$. With Fubini's theorem we obtain

$$
\mathcal{L}\left[\hat{g}_{n}\right](\lambda)=\int_{0}^{\infty} e^{-\lambda t} \hat{g}_{n}(t) d t=\frac{1}{2 \pi} \int_{-n}^{n} F(i s) \int_{0}^{\infty} e^{(i s-\lambda) t} d t d s=-\frac{1}{2 \pi} \int_{-n}^{n} \frac{F(i s)}{i s-\lambda} d s .
$$

Let $C$ be the line $[-n i, n i]$ added by the right-hand side semicircle from $n i$ to $-n i$. If $n$ is large enough, $\lambda$ lies in the interior of $C$, and Cauchy's formula implies $\int_{C} \frac{F(z)}{z-\lambda} d z=$ $-2 \pi i F(\lambda)$, where the contour integral is taken clockwise. One can show that the integral along the semicircle (denoted by $\tilde{C}$ ) vanishes as $n \rightarrow \infty$. Indeed, along the semicircle $\tilde{C}$, one has $\left|\frac{F(z)}{z-\lambda}\right| \leq \sup _{|z|=n}|F(z)| \frac{1}{n-\lambda}$, hence $\left|\int_{\tilde{C}} \frac{F(z)}{z-\lambda} d z\right| \leq \pi \frac{n}{n-\lambda} \sup _{|z|=n}|F(z)|$ goes to 0 . Therefore, $\mathcal{L}[\hat{g}](\lambda)=\lim _{n} \mathcal{L}\left[\hat{g}_{n}\right](\lambda)=F(\lambda)$. Since the Laplace transform is injective, this shows that $\hat{g}(t)=g(t)$, for $t>0$.

3. One can show that the function $\hat{g}$ is causal, i.e. it satisfies $\hat{g}(t)=0$ for all $t<0$ (see Lemma A.1 in appendix). This implies that we can determine $\hat{g}(t)$ from the real part of its Fourier transform; more precisely, for $t>0$ we have $\hat{g}(t)=\lim _{n \rightarrow \infty} \frac{2}{\pi} \int_{0}^{n} \operatorname{Re}(F(i s)) \cos (t s) d s$ (see Section 2.2 in [9]) and hence (17).

We now proceed by showing that the function $Q$ satisfies some smoothness properties in $x$. We first prove that $g$ is absolutely continuous. For this we define, for all $z \in D$

$$
\Gamma(z)=z F(z)-c
$$

where $c=\frac{\sigma_{2}}{\sigma_{1}+\sigma_{2}}$. Notice that $\Gamma(-i s)=\overline{\Gamma(i s)}$ for $s \in \mathbb{R}$, and that $\Gamma$ is holomorphic on $D$. Moreover, $\operatorname{Re}(\Gamma(i t))$ and $\operatorname{Im}(\Gamma(i t))$ converge monotonically to zero as $\mathbb{R} \ni|t| \rightarrow \infty$. Therefore, we can define for all $t \neq 0$

$$
\begin{aligned}
\gamma(t) & =\lim _{n} \frac{1}{2 \pi} \int_{-n}^{n} \Gamma(i s) e^{i t s} d s \\
& =\lim _{n} \frac{1}{\pi} \int_{0}^{n}[\operatorname{Re}(\Gamma(i s)) \cos (t s)-\operatorname{Im}(\Gamma(i s)) \sin (t s)] d s .
\end{aligned}
$$

Lemma 3.2. The function $g$ satisfies $g(t)=c+\int_{0}^{t} \gamma(s) d s$, for all $t>0$. Moreover, $g$ is non-increasing. 
Proof. We first show that $g$ is absolutely continuous on $(0, \infty)$ and that $\gamma$ is its derivative. To this end let $0<\delta<T$ and $\gamma_{n}(t)=\frac{1}{2 \pi} \int_{-n}^{n} \Gamma(i s) e^{i t s} d s$. Observe that

$$
\int_{\delta}^{T} \gamma(t) d t=\lim _{n} \int_{\delta}^{T} \gamma_{n}(t) d t=\lim _{n} \frac{1}{2 \pi} \int_{-n}^{n} \int_{\delta}^{T} \Gamma(i s) e^{i s t} d t d s=\lim _{n} \frac{1}{2 \pi} \int_{-n}^{n} \frac{\Gamma(i s)}{i s}\left(e^{i s T}-e^{i s \delta}\right) d s .
$$

Since $\frac{\Gamma(i s)}{i s}=F(i s)-\frac{c}{i s}$, we further obtain

$$
\begin{aligned}
\int_{\delta}^{T} \gamma(s) d s & =\hat{g}(T)-\hat{g}(\delta)-\lim _{n} \frac{1}{2 \pi} \int_{-n}^{n} \frac{c}{i s}\left(e^{i s T}-e^{i s \delta}\right) d s \\
& =g(T)-g(\delta) .
\end{aligned}
$$

Since $\delta$ and $T$ are arbitrary, this shows that $g$ is absolutely continuous on $(0, \infty)$ with $g^{\prime}=\gamma$.

Next let $a<b<0$. With similar arguments as above we obtain $\int_{a}^{b} \gamma(t) d t=0$. Therefore, $\gamma(t)=0$ for all $t<0$. This further entails that $\gamma(t)=\gamma(t)+\gamma(-t)$ and hence

$$
\gamma(t)=\lim _{n} \frac{2}{\pi} \int_{0}^{n} \operatorname{Re}(\Gamma(i s)) \cos (t s) d s=\lim _{n} \frac{2}{\pi} \int_{0}^{n}-\operatorname{Im}(\Gamma(i s)) \sin (t s) d s,
$$

for $t>0$. Observe that $\operatorname{Im}(\Gamma(i s))=\operatorname{Im}(i s F(i s))=\operatorname{Re}(s F(i s))$. It is straightforward to show that $\operatorname{Re}(s F(i s)) \geq 0$. Together with Equation (19) this entails that $\gamma$ is nonpositive. Consequently, $g$ is non-increasing.

Since $g$ is absolutely continuous, we can recover from its Laplace transform the righthand side limit at zero; more precisely, we have $\lim _{\lambda \rightarrow \infty} \lambda \mathcal{L}[g](\lambda)=g(0+)$ (see e.g. [2] or [3]). Observe that $\lim _{\lambda \rightarrow \infty} \lambda \mathcal{L}[g](\lambda)=\lim _{\lambda \rightarrow \infty} \lambda F(\lambda)=c$. Consequently, we have $g(t)=c+\int_{0}^{t} \gamma(s) d s$, for $t>0$.

Corollary 3.3. The function $\gamma$ is integrable on $\mathbb{R}_{+}$and its Laplace transform satisfies $\mathcal{L}[\gamma](\lambda)=\lambda F(\lambda)-c, \lambda>0$.

Proof. By Lemma 3.2, $g$ is decreasing and absolutely continuous; hence its derivative, $\gamma$, is integrable. The second statement follows from standard properties of Laplace transforms (see e.g. Section 1 in [2] or Theorem 8.1 in [3]).

Proposition 3.4. $Q \in \mathcal{C}^{0,2}((0, \infty) \times(0, \infty) \backslash\{1\}) \cap \mathcal{C}^{0,1}((0, \infty) \times(0, \infty))$.

Proof. Since $H(x, u)$ is twice continuously differentiable in $x \neq 1$, Equations (15) and (16) imply $Q \in \mathcal{C}^{0,2}((0, \infty) \times(0, \infty) \backslash\{1\})$.

We next show that $Q$ is once continuously differentiable w.r.t. the second variable $x$ in every point $(t, 1)$, with $t>0$. Differentiating (15) w.r.t. $x$, and applying the integration by parts formula yields, for $x<1$,

$$
\begin{aligned}
Q_{x}(t, x) & =\int_{0}^{t} g(t-u) H_{t, x}(x, u) d u \\
& =c H_{x}(x, t)+\int_{0}^{t} H_{x}(x, u) \gamma(t-u) d u .
\end{aligned}
$$


Similarly, for $x>1$ we have

$$
\begin{aligned}
Q_{x}(t, x) & =-H_{x}(x, t)+\int_{0}^{t} g(t-u) H_{t, x}(x, u) d u \\
& =-H_{x}(x, t)+c H_{x}(x, t)+\int_{0}^{t} H_{x}(x, u) \gamma(t-u) d u
\end{aligned}
$$

Now let $h_{2}(t)=\lim _{x \uparrow 1} H_{x}(t, x)$ and $h_{1}(t)=\lim _{x \downarrow 1} H_{x}(t, x)$. Notice that

$$
\begin{aligned}
h_{2}(t) & =\frac{2}{\sigma_{2} \sqrt{t}} \varphi\left(\sigma_{2} \sqrt{t} / 2\right)+\Phi\left(\sigma_{2} \sqrt{t} / 2\right), \\
h_{1}(t) & =-\frac{2}{\sigma_{1} \sqrt{t}} \varphi\left(\sigma_{1} \sqrt{t} / 2\right)+\Phi\left(-\sigma_{1} \sqrt{t} / 2\right) .
\end{aligned}
$$

By passing to the limit in Equation (20) we obtain

$$
\lim _{x \uparrow 1} Q_{x}(t, x)=c h_{2}(t)+\int_{0}^{t} h_{2}(u) \gamma(t-u) d u .
$$

Similarly, we get

$$
\lim _{x \downarrow 1} Q_{x}(t, x)=-h_{1}(t)+c h_{1}(t)+\int_{0}^{t} h_{1}(u) \gamma(t-u) d u .
$$

One can show that

$$
\begin{aligned}
\mathcal{L}\left[h_{1}\right](\lambda) & =\frac{1}{2 \lambda}-\frac{1}{2 \lambda} \frac{1}{\sigma_{1}} \sqrt{8 \lambda+\sigma_{1}^{2}} \\
\mathcal{L}\left[h_{2}\right](\lambda) & =\frac{1}{2 \lambda}+\frac{1}{2 \lambda} \frac{1}{\sigma_{2}} \sqrt{8 \lambda+\sigma_{2}^{2}}
\end{aligned}
$$

Observe that $\mathcal{L}[\gamma]=-c-\frac{\mathcal{L}\left[h_{1}\right]}{\mathcal{L}\left[h_{2}\right]-\mathcal{L}\left[h_{1}\right]}$. A straightforward computation shows that the Laplace transform in $t$ of the right-hand side of (22) is equal to the Laplace transform of the right-hand side of (21). Since the Laplace transforms coincide, it must hold true that $\lim _{x \uparrow 1} Q_{x}(t, x)=\lim _{x \downarrow 1} Q_{x}(t, x)$.

\section{Proof of Theorem 1.1}

In this section we prove Theorem 1.1 via classical verification. To this end observe that the HJB equation associated to problem (1) is given by

$$
v_{t}(t, x)-1_{[1, \infty)}(x)-\frac{1}{2} x^{2} \sup _{u \in\left[\sigma_{1}, \sigma_{2}\right]} u^{2} v_{x x}(t, x)=0,
$$

with initial condition $v(0, x)=1_{[1, \infty)}(x)$. 
Proof of Theorem 1.1. Let $w(t, x)=\int_{0}^{t} Q(s, x) d s$. Lemma 3.4 implies that $w \in \mathcal{C}^{1,2}((0, \infty) \times$ $(0, \infty) \backslash\{1\}) \cap \mathcal{C}^{1,1}((0, \infty) \times(0, \infty))$.

Since $w(t, x)$ grows at most linearly in $t$, we can define the Laplace transform $\bar{w}(\lambda, x)=$ $\int_{0}^{\infty} e^{-\lambda t} w(t, x) d t$. Notice that with Fubini's theorem we obtain

$$
\bar{w}(\lambda, x)=\int_{0}^{\infty} Q(s, x) \int_{s}^{\infty} e^{-\lambda t} d t d s=\frac{1}{\lambda} G(\lambda, x),
$$

where $G$ is defined by $(10)$. For every $\lambda \in(0, \infty)$ the function $G(\lambda, \cdot)$ solves the ODEs (8) and (9). Therefore, $\bar{w}(\lambda, \cdot)$ satisfies

$$
\begin{gathered}
-\lambda \bar{w}(\lambda, x)+\frac{1}{\lambda}+\frac{1}{2} \sigma_{1}^{2} x^{2} \bar{w}_{x x}(\lambda, x)=0, \quad x>1, \\
-\lambda \bar{w}(\lambda, x)+\frac{1}{2} \sigma_{2}^{2} x^{2} \bar{w}_{x x}(\lambda, x)=0, \quad x \in(0,1) .
\end{gathered}
$$

Observe that $\mathcal{L}\left[w_{t}(\cdot, x)\right](\lambda)=\lambda \bar{w}(\lambda, x)$ and $\mathcal{L}\left[w_{x x}(\cdot, x)\right](\lambda)=\bar{w}_{x x}(\lambda, x)$. Since the Laplace transform is injective, Equations (24) and (25) imply that $w(t, \cdot)$ satisfies

$$
\begin{aligned}
& -w_{t}(t, x)+1+\frac{1}{2} \sigma_{1}^{2} x^{2} w_{x x}(t, x)=0, \quad x>1, \\
& -w_{t}(t, x)+\frac{1}{2} \sigma_{2}^{2} x^{2} w_{x x}(t, x)=0, \quad x \in(0,1) .
\end{aligned}
$$

Note that $w_{t}(t, x) \in[0,1]$ for all $(t, x) \in \mathbb{R}_{+} \times \mathbb{R}_{+}$. Thus, Equation (26) entails $w_{x x}(t, x) \leq$ 0 for $x>1$, and Equation (27) yields $w_{x x}(t, x) \geq 0$ for $x \in(0,1)$. In other words, $w(t, \cdot)$ is concave on $[1, \infty)$ and convex on $(0,1)$. This further implies that $w$ satisfies the HJB equation (23) for all $(t, x) \in \mathbb{R}_{+} \times \mathbb{R}_{+} \backslash\{1\}$.

The proof that $w$ coincides with the value function $v$ and that $\eta$ is optimal follows now from a standard verification argument. To this end notice that we can apply the Itô formula to $w$, because the second derivative exists Lebesgue-a.e.

Finally, Equation (5) follows from Corollary 2.4.

Remark 4.1. Theorem 1.1 implies that the function $w$ is not twice continuously differentiable in $x$, at any point $(t, 1)$ with $t>0$. Indeed, since $w$ satisfies $(26)$ and $(27)$, we have

$$
\lim _{x \downarrow 1} w_{x x}(t, x)=\frac{2}{\sigma_{1}^{2}}(Q(t, 1)-1), \text { and } \lim _{x \uparrow 1} w_{x x}(t, x)=\frac{2}{\sigma_{2}^{2}} Q(t, 1) .
$$

If $w_{x x}$ was continuous in $(t, 1)$, then $\frac{2}{\sigma_{1}^{2}}(Q(t, 1)-1)=\frac{2}{\sigma_{2}^{2}} Q(t, 1)$. This would imply $Q(t, 1)=\frac{\sigma_{2}^{2}}{\sigma_{2}^{2}-\sigma_{1}^{2}}>1$, which is not possible, since $Q(t, 1)$ is a probability.

\section{A. Appendix}

We give here the proof that the function $\hat{g}$ defined by (18) is causal. We remark that the proof uses standard arguments that can be found e.g. in [2]. 
Lemma A.1. For $t<0, \hat{g}(t)=0$.

Proof. Let $t<0$. Observe that the function $z \mapsto e^{z t} F(z)$ is holomorphic on $D$. Let $C_{R}$ be the line $[-R i, R i]$ added by the right-hand side semicircle $\Gamma_{R}$ from $R i$ to $-R i$. Then

$$
\int_{C_{R}} e^{z t} F(z) d z=0
$$

To prove that $\hat{g}$ is causal, it is enough to prove that $\lim _{R \rightarrow \infty} \int_{\Gamma_{R}} e^{z t} F(z) d z=0$. On $\Gamma_{R}$, one has $\left|e^{z t}\right|=e^{t R \cos \theta}$. Moreover, for all $\epsilon>0$, there exists $R$ large enough such that $|F(z)|<\epsilon$, for $z \in \Gamma_{R}$. Then

$$
\int_{\Gamma_{R}} e^{z t} F(z) d z \leq 2 R \epsilon \int_{0}^{\frac{\pi}{2}} \exp (R t \cos \theta) d \theta
$$

As $t<0$ and $\sin (\theta) \geq 2 \theta / \pi$, for $\theta \in[0, \pi / 2]$, we obtain

$$
\int_{0}^{\frac{\pi}{2}} \exp (R t \cos \theta) d \theta=\int_{0}^{\frac{\pi}{2}} \exp (R t \sin \theta) d \theta \leq \frac{\pi}{2 R|t|}(1-\exp (R t))
$$

and hence the result.

\section{References}

[1] I. Althöfer, M. Beckmann, and F. Salzer. On some Random Walk Games with Diffusion Control. Preprint, 2015.

[2] G. Demengel. Transformations de Laplace: théorie et illustrations par les exemples. Ellipses Éd. Marketing, 2002.

[3] G. B. Folland. Fourier analysis and its applications. The Wadsworth \& Brooks/Cole Mathematics Series. Wadsworth \& Brooks/Cole Advanced Books \& Software, Pacific Grove, CA, 1992.

[4] M. Jeanblanc, M. Yor, and M. Chesney. Mathematical methods for financial markets. Springer Finance. Springer-Verlag London, Ltd., London, 2009.

[5] I. Karatzas and S. E. Shreve. Brownian motion and stochastic calculus, volume 113 of Graduate Texts in Mathematics. Springer-Verlag, New York, second edition, 1991.

[6] J. Keilson and J. A. Wellner. Oscillating Brownian motion. J. Appl. Probability, 15(2):300-310, 1978.

[7] J. M. McNamara. Optimal control of the diffusion coefficient of a simple diffusion process. Math. Oper. Res., 8(3):373-380, 1983. 
[8] S. Nakao. On the pathwise uniqueness of solutions of one-dimensional stochastic differential equations. Osaka J. Math., 9:513-518, 1972.

[9] A. Papoulis. The Fourier integral and its applications. McGraw-Hill, 1962.

[10] L. C. G. Rogers and D. Williams. Diffusions, Markov processes, and martingales. Vol. 2. Cambridge Mathematical Library. Cambridge University Press, Cambridge, 2000. Itô calculus, Reprint of the second (1994) edition.

[11] W. Rudin. Real and complex analysis. McGraw-Hill Book Co., New York, third edition, 1987. 